\title{
Sex Disparities in the Global Burden of Surgical Disease
}

\author{
Brittany L. Powell ${ }^{1,2} \cdot$ Rebecca Luckett $^{3,4,5} \cdot$ Abebe Bekele $^{6} \cdot$ Tiffany E. Chao ${ }^{1,7}$
}

Published online: 18 March 2020

(C) Société Internationale de Chirurgie 2020

The 2015 Lancet Commission on Global Surgery and 2015 Global Burden of Disease study provide evidence for the increasing relative burden of noncommunicable diseases in low- and middle-income countries (LMICs), including surgical conditions such as injuries, gastrointestinal diseases, and cancer $[1,2]$. While many of these conditions affect both men and women, women bear a large burden of sex-specific surgical disease.

We analyzed the 2015 Global Burden of Disease study [2] and isolated sex-specific surgical conditions. We define sex-specific conditions here in biological terms, as diseases that exclusively affect individuals with either male or female sex-specific organs, and use the term "sex" to refer to biological definitions rather than "gender." Maternal physiologic disorders that are either surgically treated or require the expertise of a surgically trained provider were included, such as hemorrhage, hypertensive disorders and eclampsia, obstructed labor, uterine rupture, fistula, abortion, miscarriage, and ectopic pregnancy, but not maternal sepsis. Pathogenic conditions including female-specific cancers, uterine fibroids, endometriosis, and genital

Tiffany E. Chao

tiffany.chao@stanford.edu

1 Stanford University School of Medicine, Stanford, CA, USA

2 Program in Global Surgery and Social Change, Boston, MA, USA

3 Beth Israel Deaconess Medical Center, Boston, MA, USA

4 Botswana Harvard AIDS Initiative Partnership, Gaborone, Botswana

5 Department of Obstetrics and Gynaecology, University of Botswana, Gaborone, Botswana

6 University of Global Health Equity, Kigali, Rwanda

7 Santa Clara Valley Medical Center, San Jose, CA, USA prolapse were included. Male-specific surgical pathogenic conditions such as prostate and testicular cancer, as well as benign prostatic hyperplasia were included (Table 1). These data demonstrate that comparing potentially fatal sex-specific surgical diseases has a female:male prevalence ratio of $38,038,200: 15,120,200$ or approximately $2.5: 1$ (Fig. 1). The addition of surgical disease that leads to morbidity or disability increases the female:male ratio of overall prevalence of surgical conditions to 361,590,500:119,745,500 or approximately 3:1 (Fig. 2).

We compared the disability-adjusted life years (DALYs) caused by these disorders using the WHO's DALY estimates for cancers for 2016 [3]. These estimates were not available for the surgery-specific maternal disorders and those diseases causing morbidity only; we used the Global Burden of Disease compare tool for these in 2015 [4]. Using these estimates of DALYs caused by surgical disease, the female:male ratio is 5.2 (Fig. 3). While there are certain other surgical conditions that affect men at higher rates-particularly trauma - these data demonstrate that for the sex-specific surgically treatable conditions recognized by the Global Burden of Disease study, women bear a hugely disproportionate burden.

The consequences of this burden are far-reaching. Obstetric complications and cervical cancer affect women during their reproductive years, when they are securing a future for their families and providing care for vulnerable infants and children. The resulting losses have crosscutting consequences, including financial instability, increased mortality rates for her children, loss of education, and difficulty managing the household [5]. On top of this, societal-level norms create barriers in access to care for women, which have been documented in country-level and region-level studies, such as education level, decisionmaking autonomy, cultural expectations, and employment. 
Table 1 All sex-specific surgical conditions

\begin{tabular}{|c|c|c|c|c|}
\hline & $\begin{array}{l}\text { Prevalence } \\
\text { (thousands) }\end{array}$ & $\begin{array}{l}\% \text { of all sex-specific surgical conditions } \\
\text { causing mortality (out of } 53,158.4 \text { ) }\end{array}$ & $\begin{array}{l}\% \text { of all sex-specific surgical } \\
\text { conditions (out of } 481,336 \text { ) }\end{array}$ & $\begin{array}{l}\text { DALYs } \\
\text { (thousands) }\end{array}$ \\
\hline $\begin{array}{l}\text { Maternal disorders (not } \\
\text { including sepsis) }\end{array}$ & 8232.1 & $15.5 \%$ & $1.7 \%$ & 7129 \\
\hline \multicolumn{5}{|l|}{ Female-specific cancers } \\
\hline Breast cancer & $21,361.8$ & $40.2 \%$ & $4.4 \%$ & 18,958 \\
\hline Cervical cancer & 3442.4 & $6.5 \%$ & $0.7 \%$ & 9654 \\
\hline Uterine cancer & 3827.7 & $7.2 \%$ & $0.8 \%$ & 2479 \\
\hline \multirow[t]{3}{*}{ Ovarian cancer } & 1174.2 & $2.2 \%$ & $0.2 \%$ & 5066 \\
\hline & $29,806.1$ & & $29,806.1$ & 36,157 \\
\hline & & $38,038.2$ & & \\
\hline \multicolumn{5}{|c|}{ Female-specific surgical diseases } \\
\hline Uterine fibroids & 151,115 & & $31.4 \%$ & 1550 \\
\hline Endometriosis & $10,758.2$ & & $2.2 \%$ & 4086 \\
\hline \multirow[t]{3}{*}{ Genital prolapse } & $161,679.1$ & & $33.6 \%$ & 341 \\
\hline & $323,552.3$ & & $353,358.4$ & 5977 \\
\hline & & & $361,590.5$ & 49,263 \\
\hline \multicolumn{5}{|l|}{ Male-specific cancers } \\
\hline Prostate cancer & $14,434.4$ & $27.2 \%$ & $3.0 \%$ & 6452 \\
\hline \multirow[t]{2}{*}{ Testicular cancer } & 685.8 & $1.3 \%$ & $0.1 \%$ & 647 \\
\hline & $15,120.2$ & $15,120.2$ & $15,120.2$ & 7099 \\
\hline \multicolumn{5}{|c|}{ Male-specific surgical diseases } \\
\hline \multirow{3}{*}{$\begin{array}{l}\text { Benign prostatic } \\
\text { hyperplasia }\end{array}$} & $104,625.3$ & & $21.7 \%$ & 2298 \\
\hline & $104,625.3$ & & & \\
\hline & & & $119,745.5$ & 9397 \\
\hline Total & & $53,158.4$ & 481,336 & 58,660 \\
\hline Female:male ratio & & 2.52 & 3.02 & 5.2 \\
\hline
\end{tabular}

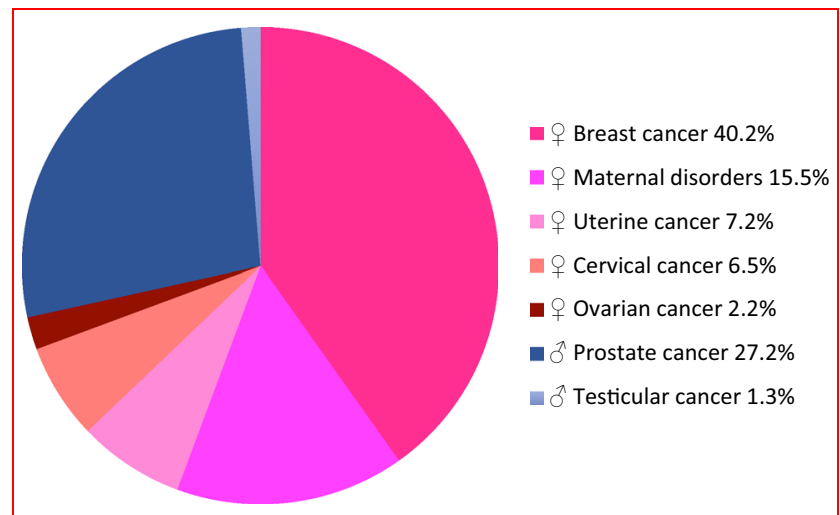

Fig. 1 Sex-specific surgical conditions causing mortality

Despite this huge disparity in sex-specific surgical burden of disease, evidence shows an inequitable distribution of access to surgical care for women, particularly in lowresource settings [6]. The third edition of Disease Control
Priorities recognized that after injuries, maternal-neonatal conditions are the second most common cause of surgically preventable deaths, responsible for 233,658 deaths per year [7]. Obstetric conditions, including maternal hemorrhage, obstructed labor, obstetric fistula, abortion, and neonatal encephalopathy, cause an estimated 56.6 million DALYs in LMICs, $37 \%$ of which would potentially be avertable by availability of quality surgical provision [8]. Breast cancer, which is estimated at 2,088,849 new cases a year, occurs in women in $99 \%$ of cases and is nearly as common as lung cancer, which is estimated at 2,093,876 new cases per year [9]. Along with cervical cancer, these diseases have become detectable earlier and are largely treatable in highincome countries (HICs), whereas they kill three times as many women as pregnancy and childbirth complications each year in LMICs, even in countries with high maternal mortality rates [10].

Primary prevention and secondary prevention of sexspecific diseases have the potential to play an important role in reducing this disparity in surgical demand; however, 
Fig. 2 All sex-specific surgical conditions causing morbidity

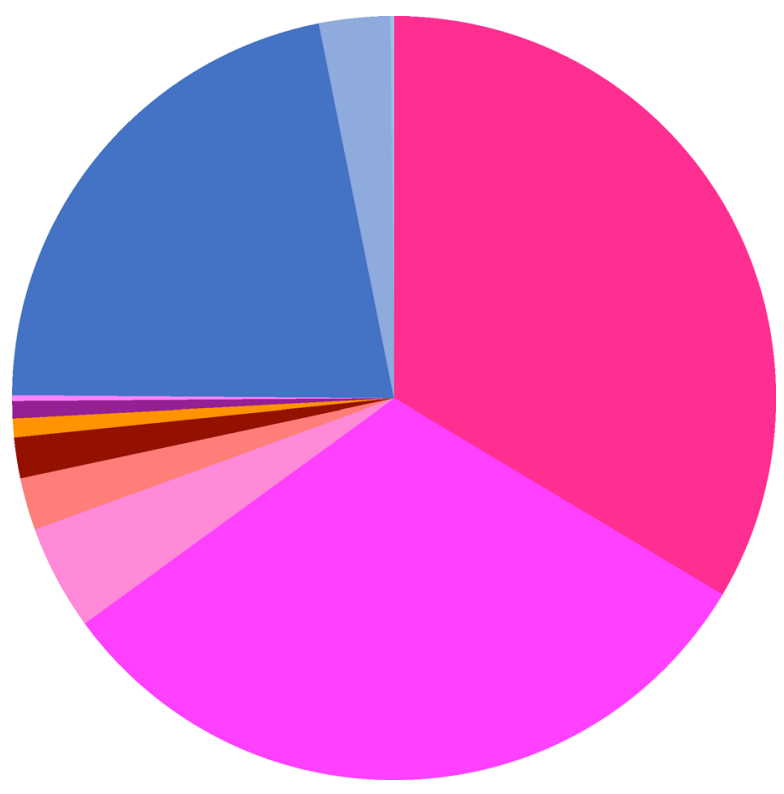

q $q$ Genital prolapse $33.6 \%$

n q Uterine fibroids $31.4 \%$

a $q$ Breast cancer $4.4 \%$

무 Endometriosis 2.2\%

- $q$ Maternal disorders $1.7 \%$

무 0 Uterine cancer $0.8 \%$

n + Cervical cancer $0.7 \%$

므우 Ovarian cancer $0.2 \%$

- 3 Benign Prostatic hyperplasia $21.7 \%$

- $\delta$ Prostate cancer 3\%

a Testicular cancer $0.1 \%$

Fig. 3 DALYs caused by sexspecific disorders

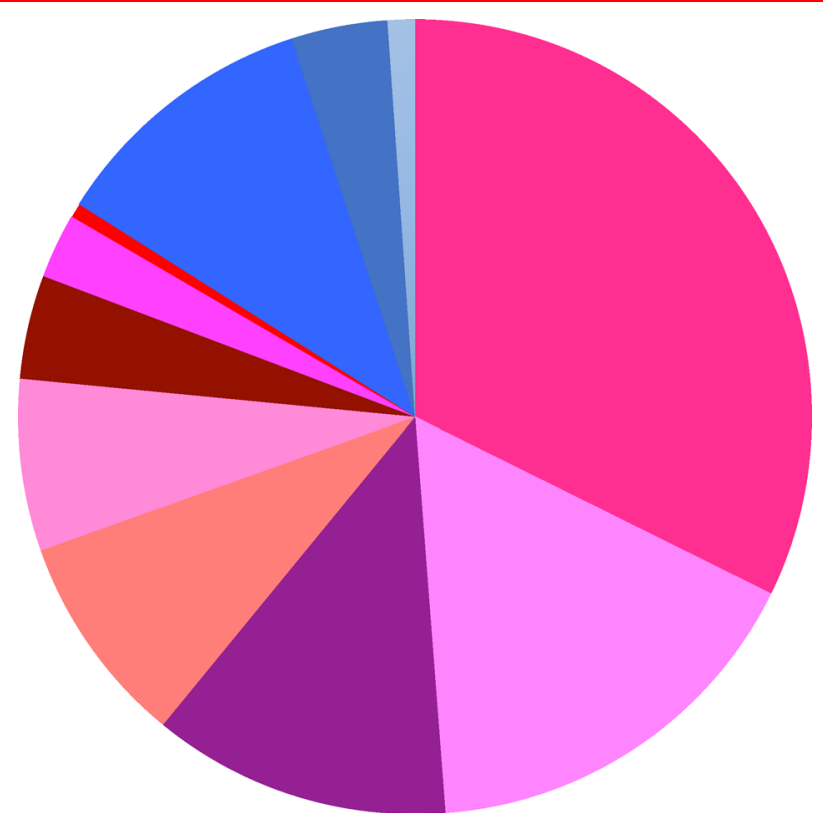

- $q$ Breast cancer

- $q$ Cervical cancer

- $q$ Maternal disorders

q Ovarian cancer

- $q$ Endometriosis

- $q$ Uterine cancer

- O Uterine fibroids

- $q$ Genital prolapse

- $\widehat{O}$ Prostate cancer

- $\widehat{\partial}$ Benign prostatic hyperplasia

- $\widehat{o}$ Testicular cancer

prevention has proven challenging to implement. Quality antenatal care mitigates the development of complications in pregnancy, but just half of pregnant women in LMICs attend the World Health Organization (WHO) minimum recommended four visits during pregnancy [11]. Likewise, prevention and early detection are cornerstones in cancer control efforts, but mammographic screening availability is so low in LMICs that the WHO encourages focusing on breast self-exams and early diagnosis of symptomatic lesions rather than routine screening programs [12]. Efforts in LMICs to expand access to cervical cancer screening methods that are feasible but which have not been shown to reduce morbidity and mortality have resulted in limited impact on the disease [13]. This is compounded by the limited reach of the human papilloma virus vaccination to an estimated $0.5 \%$ of all women in LMICs [14]. Improving universal healthcare coverage and community-based health care for early detection and appropriate referral of cases may be of utility in primary and secondary prevention efforts. 
To address the existing surgical disease burden in LMICs, an estimated 1.27 million new providers of surgical, anesthetic, and obstetric care are needed [1]. To address the burden of surgical disease that disproportionately affects women, fostering interest among medical students in specialization in surgical fields that will address female-specific surgical disease including obstetrics and gynecology and surgery is essential. Supporting trainees through mentoring and modeling sustainable careers will, in turn, address the higher burden of surgical disease that is borne by women.

Parallel to the sex disparity in surgical disease globally, there is a need to address gender disparity of trainees in surgical specialties. (Here, we use the term "gender" to refer to gender identity rather than biological sex.) In highincome countries, there has been a gender revolution in medicine, and some countries have achieved gender parity in medical student enrollment as early as 2004 [15]. There are limited data regarding the overall gender ratios in LMICs, but anecdotal reports show that despite enrollment in some medical schools reaching $45-50 \%$ female, the rate of graduating females is very well below $30 \%$. Unpublished graduate data from College of Surgeons of East, Central, and Southern Africa (COSECSA) indicate 16\% enrollment and $14 \%$ graduation record of females. It would be worthwhile to clearly identify the proportion of women entering and graduating from medical schools in various LMIC regions.

There are a number of strategies that have been helpful in high income settings for recruiting women to medicine that could be employed in LMICs. Early recognition of the gender disparity and initiation of supportive policies, enrollment quotas or enrollment gender parity, scholarships and fellowships for women, gender parity in advanced training positions, equitable pay structure, improved maternity leave, and appointment of proportionate females in leadership roles are all important considerations in recruiting and retaining female surgeons and OB/GYNs. Surgical societies such as COSECSA Women in Surgery Africa, scientific conferences, deliberate mentorship, peer role modeling, as well as virtual mentorship from international colleagues, may help provide continuing education and support for these women. In turn, we propose that the greater number of female practitioners may contribute to improving the burden of surgical disease borne by women, because female medical students often, but not always, exhibit preferences toward specialization in OB/GYN [16]. Studies in some LMICs also indicate that there is a strong patient preference for a female gynecologists and obstetricians $[17,18]$.

There are challenges, including lack of female leadership and mentorship in surgical specialties, and barriers that a lifestyle in surgery uniquely presents to women [19].
There is also a lack of sufficient schools, training positions, and programs in surgical specialties in LMICs. Technology and innovation may contribute to these solutions by linking people and ideas across borders and institutions to address these gaps, but governments and Ministries of Health can commit to more gender equity in training surgeons in the existing positions. Both women and men should be involved in the advocacy and mentorship of female trainees to help address the disparity in disease. Examining some of the successful models of support for recruiting and retaining women in surgical careers may prompt a more serious conversation about context-adapted strategies to augment female human resource capacity in LMICs. This may be one critical step to address the sex disparities in the surgical burden of disease, so that women will no longer need to bear three times the prevalence and over five times the DALYs of surgical disease.

\section{Compliance with ethical standards}

Conflict of interest Drs. Chao, Luckett, Bekele, and Powell have nothing to disclose.

\section{References}

1. Meara JG, Leather AJM, Hagander L et al (2015) Global Surgery 2030: evidence and solutions for achieving health, welfare, and economic development. Lancet 386(9993):569-624

2. Disease GBD, Injury I, Prevalence C (2016) Global, regional, and national incidence, prevalence, and years lived with disability for 310 diseases and injuries, 1990-2015: a systematic analysis for the Global Burden of Disease Study 2015. Lancet 388(10053):1545-1602

3. Organization WH. Global Summary DALY estimates 2000-2016 (2016) https://www.who.int/healthinfo/global_burden_disease/ GHE2016_DALY_Global_2000_2016_xls?ua=1. Accessed 20 Jan 2020

4. Evaluation UoWIfHMa. GBD Compare (2020) https://vizhub. healthdata.org/gbd-compare/. Accessed 20 Jan 2020

5. Pande R, Ogwang S, Karuga R et al (2015) Continuing with “..a heavy heart" - consequences of maternal death in rural Kenya. Reprod Health 12 Suppl 1:S2

6. Gyedu A, Abantanga F, Boakye G et al (2016) Barriers to essential surgical care experienced by women in the two northernmost regions of Ghana: a cross-sectional survey. BMC Womens Health 16:27

7. Debas HT, Donkor P, Gawande A, Jamison DT, Kruk ME, Mock CN (2015) Disease control priorities: essential surgery, vol 1. The World Bank, Washington

8. Higashi H, Barendregt JJ, Kassebaum NJ, Weiser TG, Bickler SW, Vos T (2015) Surgically avertable burden of obstetric conditions in low- and middle-income regions: a modelled analysis. BJOG 122(2):228-236

9. Bray F, Ferlay J, Soerjomataram I, Siegel RL, Torre LA, Jemal A (2018) Global cancer statistics 2018: GLOBOCAN estimates of incidence and mortality worldwide for 36 cancers in 185 countries. CA Cancer J Clin 68(6):394-424 
10. Ginsburg O, Bray F, Coleman MP et al (2017) The global burden of women's cancers: a grand challenge in global health. Lancet 389(10071):847-860

11. UN-DESA (2016) The millennium development goals report 2015. https://doi.org/10.18356/6cd11401-en. Accessed 4 Apr 2019

12. World Health Organization (2014) WHO position paper on mammography screening. World Health Organization, Geneva

13. Sankaranarayanan R, Nene BM, Shastri SS et al (2009) HPV screening for cervical cancer in rural India. $\mathrm{N}$ Engl $\mathrm{J}$ Med 360(14):1385-1394

14. Bruni L, Diaz M, Barrionuevo-Rosas L et al (2016) Global estimates of human papillomavirus vaccination coverage by region and income level: a pooled analysis. Lancet Glob Health 4(7): $453-\mathrm{e} 463$

15. Burton KR, Wong IK (2004) A force to contend with: The gender gap closes in Canadian medical schools. CMAJ 170(9):1385-1386
16. Ng-Sueng LF, Vargas-Matos I, Mayta-Tristan P et al (2016) Gender Associated with the Intention to Choose a Medical Specialty in Medical Students: A Cross-Sectional Study in 11 Countries in Latin America. PLoS ONE 11(8):e0161000

17. Lafta RK (2006) Practitioner gender preference among gynecologic patients in Iraq. Health Care Women Int 27(2):125-130

18. Tobler KJ, Wu J, Khafagy AM, Pier BD, Torrealday S, Londra L (2016) Gender Preference of the Obstetrician Gynecologist Provider: A Systematic Review and Meta-Analysis [1E]. Obstet Gynecol 127:43S

19. Muchemwa F, Erzingatsian K (2014) Women in surgery: factors deterring women from being surgeons in Zimbabwe. East Cent Afr J Surg 19(2):5-11

Publisher's Note Springer Nature remains neutral with regard to jurisdictional claims in published maps and institutional affiliations. 